EOMmUn:

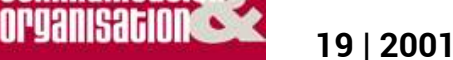

Actualité de la recherche en communication

\title{
Introduction
}

\section{Françoise Bernard}

\section{OpenEdition}

Journals

Édition électronique

URL : http://journals.openedition.org/communicationorganisation/2481

DOI : 10.4000/communicationorganisation.2481

ISSN : 1775-3546

\section{Éditeur}

Presses universitaires de Bordeaux

\section{Édition imprimée}

Date de publication : 1 mai 2001

ISSN : 1168-5549

\section{Référence électronique}

Françoise Bernard, "Introduction », Communication et organisation [En ligne], 19 | 2001, mis en ligne le 27 mars 2012, consulté le 04 mai 2019. URL : http://journals.openedition.org/ communicationorganisation/2481; DOI : 10.4000/communicationorganisation.2481

Ce document a été généré automatiquement le 4 mai 2019.

(c) Presses universitaires de Bordeaux 


\title{
Introduction
}

\author{
Françoise Bernard
}

1 En 1996, la revue consacrait le Dossier de son numéro 10 à la recherche en communication. Cinq ans après, il est apparu pertinent de donner la parole aux jeunes chercheurs afin de tracer un des possibles paysages de la recherche en communication des organisations dans sa diversité, ses évolutions et ses permanences.

2 Bien évidemment ce dossier n'a aucune prétention à l'exhaustivité, cela va de soi, ni même à une représentativité qui serait organisée sur un mode rigoureux. Il témoigne, plus modestement, de parcours de recherche de chercheurs qui sont tous titulaires d'un doctorat en SIC, soutenu récemment et souvent dans l'année qui a précédé (Durampart, Fourquet. Lépine) et qui poursuivent leurs travaux dans le cadre d'équipes reconnues.

3 Du point de vue d'une sociologie de la recherche, nous notons que les femmes continuent d'être bien représentées : quatre auteurs sur sept, ce qui était déjà le cas en 1996. Effets de sélection ou de jeu statistique représentatif de la communauté scientifique en SIC, nous donnons une préférence à la deuxième interprétation.

4 En ce qui concerne le contenu scientifique, les textes publiés sont marqués par une diversité thématique qui pourrait cependant être présentée dans le cadre de l'analyse d'une dialectique des enjeux, formes et effets d'une matérialité et d'une immatérialité de la communication des organisations.

5 Si les Technologies de l'Information et de la Communication représentent des objets de recherche légitimes et fortement légitimés notamment à partir de demandes sociales, institutionnelles et managériales, d'autres objets retiennent l'attention des jeunes chercheurs. Ce constat est réconfortant et mérite d'être souligné afin de montrer, si cela était encore nécessaire, que la communication des organisations n'est pas victime de phénomènes de mode et ne cherche pas à s'inscrire systématiquement dans des courants dominants.

6 Les questions méthodologiques sont présentes mais non traitées séparément. C'est principalement dans le cadre des études empiriques, qui relèvent parfois d'une démarche expérimentale dans l'acception des sciences humaines et sociales et plus précisément de la psychologie sociale expérimentale (Fourquet. Courbet), que sont abordées ces 
questions de méthodologie. On peut voir dans une telle évolution, un pas vers une forme de maturité de la recherche en communication des organisations. Ce qui frappe, c'est aussi une définition de la posture du chercheur qui serait pris et repris dans et par la réalité sociale qu'il induit partiellement par sa démarche auprès des acteurs de l'organisation qu'il observe. Est mise en place une boucle d'induction et contre induction qui renvoie également et par ailleurs à un des thèmes de réflexion qui a été développé par la revue (cf. $\left.\mathrm{n}^{\circ} 12,1997\right)$. Le chercheur qui induit dans le cadre d'un projet et d'une pratique de recherche est à son tour " contre induit " par la communauté professionnelle qui l'accueille (cf. notamment Durampart). La démarche du chercheur devient, pour un temps, un vécu collectif et organisationnel qui influence inévitablement les acteurs de l'organisation, le chercheur, le processus et les résultats de la recherche. Michel Durampart plaide, à sa façon, pour la reconnaissance d'un «chercheur collectif » qui émerge du processus même de la recherche empirique.

7 En ce qui concerne les objets de recherche, bien évidemment, les objets de la communication «numérique », technologique - les TIC - sont présents (Durampart; Lépine).

8 Valérie Lépine présente les résultats de trois études empiriques dont l'enjeu est notamment de repérer les écarts entre logiques de prescription d'une offre technologique et managériale, le groupware, et logiques d'«appropriation » d'acteurs individuels et collectifs dans des formes de travail renouvelées ou non.

Michel Durampart présente une réflexion autour des pratiques de médiation technologique pour les organisations du secteur social qui est nourrie de propositions pour une théorie de la médiation. Celle-ci s'inscrit dans la perspective d'une régulation sociale et organisationnelle issue d'un processus de confrontation impliquant acteurs et dispositif technique. Une telle perspective constitue une troisième voie entre celle de l'induction par l'offre technologique et celle de l'autonomie relative des usages.

10 Les deux chercheurs montrent comment les questions du lien et du sens sont revisitées, reformulées et réactualisées à travers les enjeux et pratiques de la communication technologique. Leurs travaux pourraient également s'inscrire comme autant de contributions communicationnelles pertinentes et critiques vis-à-vis du courant émergent $\mathrm{du}$ " knowledge management » dans les deux phases de développement de ce courant, celle d'une "gestion des données performantes" (Lépine), celle "de l'organisation et du partage du savoir tacite» (Durampart), (cf. Saussois. 2001, 37) ${ }^{1}$. On notera également que les travaux de Michel Durampart font état d'une reconstruction de nouvelles formes de "communication ordinaire" par les acteurs à l'occasion de la mobilisation des TIC dans les organisations. Un tel résultat bouscule des théories plus générales qui établissent le constat d'un délitement des structures de médiation traditionnelles dans les organisations qui serait provoqué par la généralisation des TIC. Plus simplement encore, Michel Durampart montre que la médiation technologique est aussi et encore une médiation humaine. Valérie Lépine, quant à elle, montre que la performance des groupware est en quelque sorte surdéterminée par des variables qui renvoient aux questions du lien et de la culture dans les équipes et que le cadrage induit par l'offre technologique et managériale peut n'avoir d'effets que sur la rhétorique, notamment la rhétorique de la coopération, ou au contraire peut influencer des pratiques soit dans le sens d'un contrôle social accru soit dans le sens d'une contribution technologique à un projet collectif, moins partagé que convergent (dans l'acception néoclassique d'une pression de l'intérêt convergent des agents économiques à un moment 
donné). Dans leurs travaux, les deux chercheurs montrent que la matérialité technologique produit et provoque aussi des effets immatériels imprévus et imprévisibles, pour lesquels Valérie Lépine présente une proposition de catégorisation (collaboration, coordination, coopération); ils montrent tout autant que les effets d'immatérialité induisent, à leur tour au moins partiellement, une "réalité " sociotechnique qui limite l'« immanence» technologique. Autrement dit, parce que les TIC sont inévitablement reprises dans et par les dynamiques de la régulation sociale et organisationnelle qu'elles contribuent à créer, il y a indétermination relative.

11 À un méta niveau, celui d'une approche comparée des discours communicationnels savants, ces deux exemples montrent que les jeunes chercheurs, en conduisant des études empiriques localisées, font preuve d'une liberté de pensée vis-à-vis de ce qui fait parfois, en quelque sorte et par ailleurs, "sens commun » dans de nombreux papiers consacrés aux TIC et à leurs usages à travers notamment les deux courants opposés, et pourtant si proches dans leur complémentarité, de "l'utopie communicationnelle» et de la « fracture numérique ».

12 La réception est un autre objet de recherche très légitime en SIC pour les chercheurs spécialisés notamment en communication des médias. Cet objet était, jusqu'aux travaux de Marie-Pierre Fourquet, relativement peu traité en communication des organisations sur le mode expérimental - que nous distinguons des études en publicité et marketing conduites en sciences de gestion -. L'originalité du papier soumis est. là encore, de présenter des éléments pour une théorie de la réception (Fourquet, Courbet). Dans ce but, les chercheurs ont fait le pari d'une interdisciplinarité qui n'est pas seulement convoquée dans les textes mais qui est vécue en laboratoire et sur le terrain de l'expérimentation. Là aussi, nous soulignons une pratique scientifique qui va dans le sens de la maturité de la recherche en communication des organisations.

13 L'éthique est un objet qui rappelle, si cela était nécessaire, combien les objets de la communication des organisations sont partagés traditionnellement par les chercheurs et les praticiens. L'éthique dans les affaires et la communication a une histoire et des portedrapeaux, en communication, cela est vrai, par exemple, pour les relations publiques et pour le journalisme d'entreprise. Catherine Loneux reproblématise un «objet classique » en mettant ainsi en lumière une des figures des pratiques communicationnelles qui consistent notamment à mettre en scène des arguments de modernité qui se conjuguent à des phases de cryptomnésie. Par ailleurs, l'auteur montre que la recomposition des métiers et des pratiques professionnelles ne concerne pas seulement les «e activités » qui accompagnent le développement des TIC (e documentation, e commerce, cyberjournalisme. e formation, etc.), mais que cette recomposition est également opératoire pour d'autres activités « plus traditionnelles » avec l'apparition des spécialités de « déontologue » et d'« expert en éthique».

14 Effets de matérialité-immatérialité, jeux d'ombre et de lumière autour d'un débat scientifique globalement ajourné en SIC, celui de la médiologie. Sylvie Bourdin, dans un texte à quatre mains avec Daniel Bougnoux, prend le risque d'ouvrir ce débat pour la communication des organisations. Les questions de la traçabilité, des corps intermédiaires/des formes de travail collectif ont une forte présence dans les organisations, notamment au travers des programmes qualité/certification qui ont, de ce point de vue, servi de révélateurs. La tentative de déplacement de la «médiologie » vers les organisations, outre son originalité, a aussi pour mérite d'attirer l'attention sur un ensemble de propositions - la médiologie - qui provoque à ce jour plus de stratégies 
d'évitement que d'échange dans la communauté des chercheurs en Information et Communication. Paradoxe à multiples dimensions qui ne sera pas dévoilé ici, puisque le débat est réouvert sur le front de la communication des organisations...

15 Les textes présentés témoignent, selon nous, d'une avancée vers la maturité des pratiques de recherche en communication des organisations qui est marquée par :

16 - le refus de toute théorie générale et la construction de théorisations locales liées à la conduite d'études empiriques.

17 - une réflexion méthodologique étroitement associée à la conduite d'études empiriques significatives,

18 - une diversité d'objets: objets émergents, ou objets antérieurement légitimés et reproblématisés,

19 - une approche par l'interdisciplinarité « vécue » plus que « déclarée »,

20 - la présentation d'hypothèses à contre courant de la rumeur dominante, la volonté d'ouvrir des débats laissés entre parenthèses.

21 Le paysage de la recherche construit par la nouvelle génération des chercheurs permet de mesurer le chemin parcouru ces dernières années dans la structuration des activités de recherche. Nous avons parlé de "maturité ", si elle reste relative comparativement à d'autres disciplines des sciences humaines et sociales, des avancées significatives peuvent cependant être observées et s'il s'avère qu'elles conduisent à des impasses, cela fait partie du processus même d'une recherche vivante. Souhaitons que la vitalité mise en lumière soit accompagnée d'une réactivité chez les lecteurs qui se traduirait par des échanges et des débats autour du Dossier proposé. Lecteurs à vos plumes - à vos claviers - pour des auteurs à l'écoute - sur leurs écrans-...

\section{NOTES}

1. Saussois J-M., «Organiser le partage du savoir ». Sciences Humaines, mars-avril 2001.36-37. 